\title{
Factores psicosociales relacionados con el consumo doméstico de agua en una región semidesértica
}

\author{
Rocío Haydee Arreguín-Moreno, M Des Reg,(1) Rosario Román-Pérez, D en C,(2) Jesús Francisco Laborín-Álvarez, D en C, (2) \\ José Luis Moreno, MD, ${ }^{(3)}$ Elba Abril Valdez, MSP, ${ }^{(2)}$ Blanca Valenzuela, D en C Educ. ${ }^{(1)}$
}

\begin{abstract}
Arreguín-Moreno RH, Román-Pérez R, Laborín-Álvarez JF, Moreno JL, Abril Valdez E, Valenzuela B. Factores psicosociales relacionados con el consumo doméstico de agua en una región semidesértica. Salud Publica Mex 2009;5 I:32I-326.
\end{abstract}

\section{Resumen}

Objetivo.Analizar los factores psicosociales que intervienen en el consumo doméstico del agua en Hermosillo, Sonora, región del noroeste de México caracterizada por ser semidesértica. Para ello se trabajó con una muestra seleccionada de forma intencional de 198 personas (79 hombres y 119 mujeres) de una institución de educación superior, con base en las teorías de la acción razonada y la autoeficacia. Material y métodos. Se aplicó una escala que incluyó los factores psicosociales analizados, con opciones tipo Likert. Resultados. La media de edad fue de 35 años. La relación entre los factores fue positiva y moderada, en particular entre intención-norma, creencias-intención, creencias-autoeficacia, actitud-intención, actitud-autoeficacia y autoeficacia-intención. Conclusión. Si bien las características de la muestra no permiten generalizar resultados, el estudio muestra la utilidad de los factores psicosociales analizados y sugiere la posibilidad de incorporarlos en programas educativos para el cuidado del agua en el hogar.

Palabras clave: consumo de agua; actitudes; prácticas; autoeficacia; México

\author{
Arreguín-Moreno RH, Román-Pérez R, Laborín-Álvarez JF, \\ Moreno JL,Abril Valdez E, Valenzuela B. \\ Psychosocial factors associated \\ with domestic water consumption \\ in a semidesert region. \\ Salud Publica Mex 2009;5 I:321-326.
}

\begin{abstract}
Objective. To analyze psychosocial factors related with domestic water consumption in Hermosillo, Sonora, a semidesert region in northwestern Mexico. An intentionally selected sample of 198 people (79 men and 119 women) from a higher education institution was used to evaluate the theories of rational action and self-efficacy. Material and Methods.A scale with the psychosocial factors to be analyzed and Likert-type items was applied. Results:The age mean was 35 years old.A positive moderate association was found between the diverse factors, particularly between intentionnorm, beliefs-intention, beliefs-self-efficacy, attitude-intention, attitude-self-efficacy, and self-efficacy-intention. Conclusions. Despite the fact that the sample characteristics do not allow results to be generalized, the study shows the usefulness of the psychosocial factors that were analyzed and suggests the possibility of including them in educational programs that promote careful water use at home.
\end{abstract}

Key words: water consumption; attitudes; practices; selfefficacy; Mexico

(I) Universidad de Sonora. Sonora, México.

(2) Centro de Investigación en Alimentación y Desarrollo, AC. Coordinación de Desarrollo Regional. Sonora, México.

(3) El Colegio de Sonora. Sonora, México.

Fecha de recibido: 2 de septiembre de 2008 - Fecha de aceptado: 12 de mayo de 2009 Solicitud de sobretiros: Dra. Rosario Román Pérez. Centro de Investigación en Alimentación y Desarrollo,AC. Carretera a la Victoria Km. 0.6. 83000 Hermosillo, Sonora, México.

Correo electrónico: rroman@ciad.mx 
$\mathrm{E}^{\mathrm{l}}$ agua es un elemento indispensable para la salud pública, sea por su disponibilidad o por su saneamiento. ${ }^{1}$ Sin embargo, su disponibilidad y acceso para el consumo humano es uno de los retos principales que deben enfrentarse en el siglo XXI. Una sexta parte de la población no tiene posibilidad de adquirir agua potable (1100 millones) y casi 40\% carece de saneamiento (2 400 millones), lo que limita la salud humana. ${ }^{1}$ El problema no sólo es la escasez de agua, sino también el pobre cuidado que se le da a la que hay.

Según el Informe de Desarrollo Humano, ${ }^{2}$ el acceso al agua está concebido como un derecho que comprende la salud, un medio ambiente sano, la alimentación y la vivienda adecuada. En ese documento se indica que una persona necesita al menos 20 litros diarios para satisfacer sus necesidades básicas. Sin embargo, la distribución de tal recurso es inequitativa entre los países y los consumos varían según sea el nivel de desarrollo que alcancen. En Estados Unidos de América, el consumo doméstico es de 596 litros por habitante al día y en Canadá de 766 L/hab/día, mientras que en países latinoamericanos, como México, el consumo es de 382 L/hab/día. En otras naciones, como Brasil, con menor acceso al agua, el consumo per cápita por año es de 192 L/hab/día, o bien países como Bangladesh, donde la situación es precaria, el consumo no rebasa los $44 \mathrm{~L} /$ hab/ día. ${ }^{3}$

Por su parte, en algunas ciudades mexicanas, como Monterrey, Nuevo León, se observan consumos de 185 L de agua por habitante al día (L/hab/ día), en el Distrito Federal y Tijuana, Baja California, son de 200 L/ hab / día y en Culiacán, Sinaloa, de 240 L/ hab/ día. En la ciudad de Hermosillo, Sonora, donde se realizó el presente estudio, se consumen 321 a $375 \mathrm{~L} /$ hab/ día, ${ }^{4}$ pese a que se encuentra ubicada en una región semidesértica y con problemas de disponibilidad de agua. Por la misma razón, durante el año 2006 las autoridades racionaron su uso en algunas colonias para optimizar el recurso. ${ }^{4}$

Algunos estudios se han elaborado a partir del modo en que los factores psicosociales afectan el comportamiento de ahorro o el desperdicio de agua. Tales factores se han analizado al tomar como base la teoría que desarrollaron Ajzen y Fishbein, que incluye las creencias, las actitudes y la norma subjetiva, entre otros. ${ }^{5}$ La teoría de la acción razonada, como se la conoce, emplea la predicción y la comprensión del comportamiento humano para resolver problemas y tomar decisiones. Se basa en la presuposición de que las personas son casi siempre racionales y hacen uso sistemático de la información o el conocimiento que tienen a su disposición. Asimismo, se asume que consideran las implicaciones de sus acciones antes de decidir si participan o no en un determinado comportamiento.
La teoría toma en cuenta la "intención" como un determinante inmediato de la acción. La intención depende de dos factores, uno de naturaleza personal, que es la actitud, referido a la evaluación positiva o negativa que hace el individuo acerca de si es bueno o malo ejecutar cierto comportamiento. El otro refleja la influencia social y se conoce como norma subjetiva; esto es, la persona responde de manera selectiva a ciertos grupos que son significativos porque representan un punto ideal con el que se compara y a través del cual evalúa lo apropiado de su conducta.

A los factores actitud y norma subjetiva les anteceden el conocimiento y las creencias. El primero hace referencia a toda la información que la persona adquiere y considera verdadera y el segundo es la evaluación del uso de tal conocimiento y la demostración de su funcionamiento tal y como estaba previsto (figura 1).

La teoría de la acción razonada se ha utilizado para estudiar conductas complejas, por ejemplo las actitudes hacia la enfermedad mental. GarcíaSilberman comprobó su funcionalidad tras evaluar los conocimientos, creencias, actitudes e intenciones hacia los enfermos mentales. Sin embargo, al igual que en otros estudios, encontró que existe discordancia entre la conducta y la intención como factor que precede al comportamiento. 6

En relación con el uso del agua, Van Vugt utilizó algunos factores como las creencias para observar conductas de ahorro de este recurso natural. ${ }^{7}$ El estudio se realizó con una población de 76 personas habitantes de Hampshire, Inglaterra. Se introdujeron además medidores en los hogares que no los tenían, lo que hizo que disminuyera de manera paulatina la cantidad de agua utilizada. Los resultados indican que las personas refieren hacer buen uso del agua, lo cual puede explicarse por la retroalimentación que se tiene del uso a través del medidor y el cobro justo por el recurso, de tal manera que se advierte lo que se gasta por el agua.

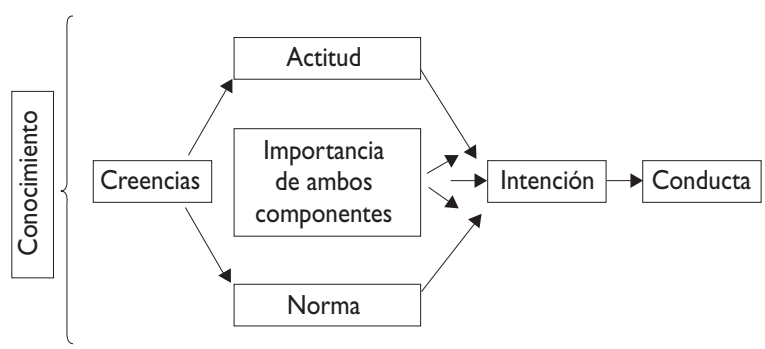

Figura I. Esquema de LA TEORÍA de LA ACCión RAZONADA (Ajzen y Fishbein, 1980). 
Otros autores, como Kaiser, Hübner y Bogner, contrastaron una nueva versión de la teoría de Azjen y Fishbein con los factores valor-creencia-norma (VBN, por sus siglas en inglés). Además, incluyeron otros factores integrados en otra modalidad de la teoría conocida como de la acción planeada (TPB, por sus siglas en inglés). Ésta comprende factores como actitud, norma subjetiva, control conductual percibido, intenciones conductuales y conducta general ecológica para explicar la conducta de conservación de los recursos naturales. ${ }^{8}$ El estudio lo realizaron con un grupo de 468 estudiantes universitarios. Los hallazgos en la aplicación del VBN mostraron que los factores valor, creencia y norma eran independientes, es decir, que uno no modificaba al otro. Por su parte, en la TPB se encontró que los factores están ligados y cuanto más positivos fueron más ecológica fue la conducta .

Craig y Garrett analizaron algunos factores como la intención y conducta hacia la conservación del agua, también a partir de la teoría de la acción razonada. ${ }^{9}$ Además, incluyeron actitudes, creencias, normas subjetivas, acciones pasadas y variables atributivas (sexo, ingreso, edad, años de residencia). De igual modo, consideraron los valores ambientales y el conocimiento acerca de la conservación del agua. Los datos se colectaron por teléfono entre 1998 y 2000 en Reno, Nevada, con 733 participantes. Los resultados mostraron que la intención se formó a partir de las actitudes y las normas y que la conducta se vincula con el conocimiento. Se ha enfatizado la importancia que tienen los valores ambientales y los patrones de conducta reforzados en el pasado.

Laborín y colaboradores seleccionaron una muestra de 437 participantes, hombres y mujeres de 18 a 52 años de edad, residentes de la ciudad de Hermosillo, Sonora, y aplicaron una escala de actitudes hacia el cuidado del agua. ${ }^{10}$ Se evaluaron seis factores: responsabilidad social, responsabilidad individual, prácticas de ahorro, locus de control, situación (consumo de agua de acuerdo con la zona geográfica) y valoración del clima. Los resultados indican que la población tiene actitudes positivas en relación con el cuidado de su consumo doméstico de agua, pero no se refleja en sus prácticas. Al igual que en los estudios ya mencionados, los factores psicosociales analizados no son determinantes por sí solos del comportamiento.

Ante la disparidad de resultados sobre los factores psicosociales y su relación con el consumo doméstico del agua, es pertinente evaluar otros factores, además de los planteados por Azjen y Fishbein, como el de la autoeficacia de Bandura.,11 Ello se debe a que este comportamiento exige no sólo tener conocimientos y predisposición a la acción, evaluados con anterioridad, sino también sentirse capaz de realizarlo. De acuerdo con Bandura, la autoeficacia se refiere a la forma en que la gente juzga sus capacidades y el efecto que la motivación tiene sobre su comportamiento. Se centra en la eficacia personal para ejercer algún control sobre sucesos que afectan las decisiones. La autoeficacia se deriva del autoconocimiento, esto es, la persona evalúa y conoce las situaciones en las que se desempeña con éxito y en las que no. ${ }^{11}$

Es por esa razón que el objetivo del presente trabajo es analizar los factores psicosociales que intervienen en el consumo doméstico de agua en Hermosillo, Sonora, una región semidesértica del noroeste de México. Para ello se optó por trabajar con una muestra seleccionada intencionalmente por su alto nivel de escolaridad, tras presuponer que contaba con información sobre la situación de escasez de agua en su localidad. Se recurrió a la teoría de la acción razonada y se agregó la de la autoeficacia por las razones ya señaladas. ${ }^{5,11}$ Como hipótesis se presupuso que en la población estudiada el consumo doméstico de agua se relacionaba con factores psicosociales, como la intención, el conocimiento, las creencias, las actitudes, la norma subjetiva y la autoeficacia.

\section{Material y métodos}

El estudio se llevó a cabo con el personal de una institución de educación superior en Hermosillo, Sonora, cuyo comité de ética evaluó y aprobó el protocolo de investigación. La muestra estudiada fue de 198 personas. La escolaridad promedio fue de maestría y el levantamiento de datos se realizó en el mes de marzo de 2006, cuando apenas iniciaba la temporada de calor en la región.

Las características de la población de estudio fueron: $61 \%$ de mujeres y $39 \%$ de varones. La media de edad fue de 35 años, con un rango etario de 22 a 58 años. La escolaridad se concentró en licenciatura (33\%) y posgrado $(64 \%)$. Las personas con pareja representaban $44 \%$ del total de la población.

A partir de una escala de actitudes evaluada de forma previa se elaboró una nueva versión con los enunciados de mayor peso factorial (mayor de 0.30). ${ }^{10}$ Se incluyó el consumo de agua para higiene personal: lavado de dientes, ducha y limpieza del hogar, como regado de la acera, lavado de utensilios de cocina y ropa. También se preguntó cuán importante era la opinión de la familia (padres, parejas, hermanos) de la persona participante (norma subjetiva) en cuanto al modo de utilizar el agua, tanto como sobre el racionamiento de ésta instituido por el gobierno municipal.

Para evaluar la autoeficacia se elaboraron enunciados tales como "puedo lavarme los dientes sólo con 
un vaso de agua", "puedo bañarme en cinco minutos", entre otros aspectos, con opciones de respuesta diversas, desde la categoría cinco que representaba muy capaz de poder hasta la uno que indicaba la incapacidad de poder hacerlo. Para los conocimientos se utilizaron respuestas dicotómica que tenían como opciones cierto y falso y para el resto de los factores las opciones fueron tipo Likert de cinco puntos, donde cinco es la categoría mayor y uno la menor. La escala contenía además datos de identificación, como el sexo, la edad o la escolaridad, entre otros más.

\section{Procedimiento}

Antes de aplicar la escala para que la respondiera la misma persona en su área de trabajo se obtuvo el consentimiento informado de cada participante. Se solicitó además el último recibo de cobro del agua para comparar el uso del recurso con las respuestas sobre las prácticas de consumo que se plantearon. Se consideró un promedio total de 15 minutos para responder.

\section{Secuencia de análisis}

El análisis se realizó con el programa estadístico SPSS (Statistic Package for Social Sciences, versión 12.0). Asimismo, se evaluó la confiabilidad (valores alfa de Cronbach) de los factores. Se compararon las respuestas sobre consumo de agua tras analizarlas por percentiles. Se formó un grupo de consumo alto de agua con las personas que respondieron haber regado la acera o bañarse en más de cinco minutos, entre otras respuestas. En caso contrario se integraron en un grupo de consumo bajo. Con posterioridad se efectuó un análisis de varianza (ANOVA) de los factores en conjunto para observar las diferencias entre los grupos de consumo de agua alto y bajo. Se hicieron correlaciones de Pearson entre los factores para obtener los valores de asociación entre éstos.

\section{Resultados}

Se obtuvieron rangos de consumo de agua a partir de los registros del recibo, según los metros cúbicos consumidos por percentiles $(25,50$ y 75$)$. El primer rango se ubicó en una media de 2.41 habitantes por hogar. El segundo rango fue de 3.00, el tercero de 3.55 y el cuarto de 4.05. Es decir, a medida que aumentaba la media de habitantes por hogar el rango de consumo fue mayor (cuadro I), valores coherentes respecto de la cantidad de integrantes de las familias. Las personas que vivían solas tuvieron un consumo más alto de agua (cuadro II).

A continuación se realizaron ANOVA con los grupos de consumos alto y bajo y se observaron diferen-

\section{Cuadro I}

RANGos de CONSUMO SEgÚN LOS HABITANTES en el hogar. Hermosillo, Sonora, México, 2006

\begin{tabular}{cccccc}
$\begin{array}{c}\text { No.de } \\
\text { rangos }\end{array}$ & $\begin{array}{c}\text { Rango en } \mathrm{m}^{3} / \\
\text { familialmes }\end{array}$ & $\begin{array}{c}\text { Equivalente } \\
\text { L/hab/dia }\end{array}$ & $\begin{array}{c}\text { Integran- } \\
\text { tes }\end{array}$ & $\begin{array}{c}\text { Des- } \\
\text { viación } \\
\text { estándar }\end{array}$ & $\mathrm{N}$ \\
\hline $\mathrm{I}$ & $0-9.87$ & $0-137$ & 2.41 & 1.44 & 22 \\
\hline 2 & $9.88-14.64$ & $110-163$ & 3 & 0.96 & 22 \\
\hline 3 & $14.65-24.24$ & $137-234$ & 3.55 & 1.42 & 22 \\
\hline 4 & $24.25-42$ & $205-346$ & 4.05 & 0.57 & 22 \\
\hline
\end{tabular}

\section{Cuadro II \\ INTEGRANTES POR HOGAR Y PROMEDIO DE consumo en metros cúbicos. Hermosillo, Sonora, Méxıco, 2006}

\begin{tabular}{ccc} 
Integrantes & $\begin{array}{c}\text { Promedio de metros } \\
\text { cúbicos consumidos }\end{array}$ & $\begin{array}{c}\text { Desviación } \\
\text { estándar }\end{array}$ \\
\hline 1 & 12 & 8.04 \\
\hline 2 & 11.66 & 6.53 \\
\hline 3 & 15.16 & 6.80 \\
\hline 4 & 20.41 & 8.59 \\
\hline $5-8$ & 22.88 & 11.71 \\
\hline
\end{tabular}

cias estadísticamente significativas $(p \leq 0.05)$ entre los factores conocimiento, norma subjetiva y autoeficacia. Esto sugiere que el conocimiento sobre la situación de escasez del agua puede contribuir a que se consuma menos este líquido. De igual manera, cuando se considera la opinión de los referentes importantes, tales como los padres, pareja y hermanos, el consumo también es menor (norma subjetiva). Asimismo, percibirse capaz de utilizar racionalmente el agua (autoeficacia) se relacionó de modo directo con el consumo bajo (cuadro III).

Por último, se presentan correlaciones de Pearson en las que se compara cada factor en relación con los demás. La teoría de la acción razonada explica que cuando un factor tiene correlación de relevancia estadística, es probable que las actitudes sean positivas y exista predisposición a la acción. Por ejemplo, si en el factor creencias se obtienen correlaciones positivas estadísticamente significativas, es probable que los demás factores cambien en la misma dirección. En la población estudiada se obtuvieron relaciones positivas de importancia estadística de 0.14 a 0.42 entre los componentes: intención-norma, creencias-intención, creencias-autoeficacia, actitudintención, actitud-autoeficacia y autoeficacia-intención. También se observaron correlaciones positivas con valores bajos en el factor norma subjetiva al compararlo con actitudes e intención (cuadro IV). 
Cuadro III

ANÁLISIS DE VARIANZA, FACTORES Y CONDUCTA DE USO PARA LOS GRUPOS BAJO Y ALTO. Hermosillo, Sonora, México, 2006

\begin{tabular}{|c|c|c|c|c|}
\hline \multirow[b]{2}{*}{ Factores } & \multirow[b]{2}{*}{$F$} & \multirow[b]{2}{*}{ Significancia } & \multicolumn{2}{|c|}{ Media } \\
\hline & & & Grupo bajo & $\begin{array}{c}\text { Grupo } \\
\text { alto }\end{array}$ \\
\hline Conocimiento* & 2.59 & 0.02 & 1.45 & 1.65 \\
\hline Creencia & 1.12 & 0.34 & 4.11 & 4.96 \\
\hline Norma subjetiva* & 2.69 & 0.01 & 4.11 & 3.74 \\
\hline Actitud & 0.89 & 0.50 & 4.16 & 4.06 \\
\hline Intención & 1.81 & 0.09 & 2.06 & 2.73 \\
\hline Autoeficacia* & 5.62 & 0.00 & 3.65 & 3.43 \\
\hline$p=<0.05$ & & & & \\
\hline
\end{tabular}

Cuadro IV

Correlaciones de Pearson para los factores. Hermosillo, Sonora, México, 2006

\begin{tabular}{lcccc}
\multicolumn{1}{c}{ Factores } & Creencia & Autoeficacia & $\begin{array}{c}\text { Norma } \\
\text { subjetiva }\end{array}$ & Actitud \\
\hline Autoeficacia & $0.16^{\ddagger}$ & & & \\
\hline Norma subjetiva & 0.05 & $0.12^{\ddagger}$ & & \\
\hline Actitud & $0.36^{*}$ & 0.05 & $0.15^{\ddagger}$ & \\
\hline Intención & 0.17 & $0.42^{*}$ & $0.16^{\ddagger}$ & $0.16^{*}$ \\
\hline$* p=<.01$ & & & & \\
$\ddagger p=<.05$ & & & & \\
\hline
\end{tabular}

\section{Discusión}

Los conocimientos, la norma subjetiva y la autoeficacia fueron los factores que más se vincularon en el grupo con menor consumo de agua. ${ }^{8,9}$ Resultados similares registraron Kaiser y colegas ${ }^{8}$ para los factores norma e intenciones, que se relacionan estadísticamente con una conducta proecológica, como es el cuidado del agua. Estos resultados sugieren la incorporación en los programas de educación para el cuidado en el consumo doméstico del agua de factores tales como el conocimiento específico sobre la situación de escasez del agua, la participación de grupos de referencia importantes para las personas como los padres, hermanos o pareja (norma subjetiva), así como el énfasis en las percepciones de autovaloraciones (autoeficacia).

Por otro lado, los resultados coinciden con el estudio de Craig y Garrett, quienes encuentran que la intención se relaciona con actitudes, normas subjetivas y autoeficacia, además de que la conducta de consumo de agua se vincula con el nivel alto de conocimiento. ${ }^{9}$ En cuanto a la utilidad de la teoría de la acción razonada y el factor de autoeficacia, el estudio mostró la relación entre los factores psicosociales analizados y el consumo del agua. Lo anterior ratifica lo encontrado por GarcíaSilberman sobre la importancia de estos factores, aunque señala que no siempre existe una relación lineal positiva entre la conducta y la intención. ${ }^{6}$

Se puede concluir que el consumo doméstico de agua se vincula sobre todo con el nivel de conocimiento, la norma subjetiva y la autoeficacia de las personas. Sin embargo, una limitante de este estudio es el nivel educativo de la población estudiada, que no es el común denominador del resto de la población. De ahí parte la pertinencia de explorar la forma en que se comportan los factores evaluados en este trabajo en relación con otro tipo de poblaciones de distintos niveles educativos. No menos importante es aplicar un sistema de retroalimentación, ya que en la actualidad $77 \%$ de la población cuenta con medidores de agua en sus hogares ${ }^{4}$ por lo que es necesario incrementar la cobertura. Ello permitirá que toda la población conozca su consumo de agua y lo corrobore con el cobro de una tarifa justa y con criterios claros, tal y como lo sugieren los estudios Ávila y Van Vugt. ${ }^{5,12}$

\section{Referencias}

I. Carabias J, Landa R, Collado J, Martínez P. Situación mundial del agua. Agua, medio ambiente y sociedad. México, DF: El Colegio de México, 2005:|4-2|.

2. Informe de Desarrollo Humano. Más allá de la escasez: poder, pobreza y crisis mundial del agua [serie en internet] 2006 [consultado: 2007, febrero 29]. Disponible en: http//Unda.org.

3. Oekstra A, Chapagain A. Water footprints of nations: water use by people as a function of their consumption patterns. Water Resource Management, 21:3I-48. [serie en internet] 2007 [consultado: 2008, agosto 25]. Disponible en: http://www.waterfootprint.org/Reports/Hoekstra and_Chapagain_2007.pdf

4. Pineda PN. Las tarifas y la autosuficiencia financiera, la equidad y la conservación del agua: un estudio comparativo de las tarifas urbanas de agua potable en Sonora. En: Pineda PN (comp). La búsqueda de la tarifa justa. México: El Colegio de Sonora, 2006: I I5-I 28.

5.Ajzen I, Fishbein M. Understanding attitudes and predicting social behavior. Englewood Cliffs: Prentice-Hall, 1980.

6. García-Silberman S. Un modelo explicativo de la conducta hacia la enfermedad mental. Salud Publica Mex 2002; 44(4):289-296.

7.Van VM. Solving natural resource dilemmas through structural change: the social psychology of metering water use. In: Foddy M, Smithson M, Schneider S, Hogg M (eds.). Resolving social dilemmas. Sydney: Psychology Press, 2000:21-134.

8. Kaiser FG, Hübner G, Bogner FX. Contrasting the theory of planned behavior with the value-belief-norm model in explaining conservation 
behavior.] Appl Soc Psychol [serie en internet] 2005 [consultado: 2006, octubre 16]; 35(10):2150-2/70. Disponible en:

http://www3.interscience.wiley.com/journal/I I 864524I/

abstract? CRETRY $=$ I\&SRETRY $=0$

9. Craig WT, Garrett JO. Intention to conserve water: environmental values, planned behavior, and information effects. Soc Nat Res [serie en internet] [consultado: 200I, diciembre 30]; I4:889-899. Disponible en: http://www.science.oregonstate.edu/ stemmere/Documents/ Intention\%20to\%20Conserve\%20Water.pdf
I0. Laborín AJ, Córdova MA,Vera NJ,Arreguín MR, Valenzuela B. Prácticas de consumo y actitudes hacia el cuidado del agua en habitantes de una zona urbana. Psicol Sal 2004; 14(2): I89-194.

II. Bandura A. Self-efficacy. Social Foundations of thought and action: a social cognitive theory. New Jersey: Englewood Cliffs, 1986:390-449.

12. Ávila FB. La cultura del agua en la ciudad de Hermosillo, análisis de la campaña comunicativa 2003-2005 (tesis). Hermosillo, Sonora, México: El Colegio de Sonora, 2005. 